\title{
Irradiated glass and thermoluminescence yield: Dosimetric utility reviewed
}

\author{
D.A. Bradley ${ }^{\mathrm{a}, \mathrm{b}, *}$, M.U. Khandaker ${ }^{\mathrm{a}}$, Abdulaziz Alanazi ${ }^{\mathrm{b}, \mathrm{c}}$ \\ ${ }^{a}$ Centre for Biomedical Physics, Sunway University, 50603, Kuala Lumpur, Malaysia \\ ${ }^{\mathrm{b}}$ Department of Physics, University of Surrey, Guildford, Surrey, GU2 7XH, United Kingdom \\ ${ }^{\mathrm{c}}$ Medical Physics Department, Cancer Centre, Prince Mohammed Medical City, P.O. Box 2254, Sakaka Aljouf, 42421, Saudi Arabia
}

\section{A R T I C L E I N F O}

\section{Keywords:}

Thermoluminescence

Dosimetry

Doped silica

Radiation medicine

\begin{abstract}
A B S T R A C T
With numbers of efforts being made towards harnessing the thermoluminescence yield of doped glass media for dosimetric applications, predominantly in the radiotherapeutic regime, review is provided of the background to this, tracing developments leading to the present day. Included are an examination of the relative strengths of the various TLD currently on offer and that of glass fabrications, commercial Ge-doped optical fibre as well as novel fibres fabricated from Ge-doped glass. The demands that modern radiotherapeutic dose delivery systems are placing upon these passive forms of dosimetry are reviewed together with the various responses arising from current efforts. Also reviewed are the basis of the luminescence yield, citing the defect types occurring in silica, even in the absence of extrinsic dopants.
\end{abstract}

\section{Introduction}

Numerous applications in the health sector rely on controlled delivery of incident energy, present focus being on the harnessing of luminescence from irradiated glass-based dosimeters. Review is made herein of developments that have directly contributed to the thermoluminescence work that has been recently presented at ICDA- 3 by the group of Bradley, exploring prospects for future utilisation of glassbased systems. Examples of prominent challenges include: tumour therapeutics, maximising target dose while preserving surrounding healthy tissue (IAEA, 2016); radiosurgery, offering fine-beam scalpellike spatial acuity (to within $1 \mathrm{~mm}$ ); image-guided radiology interventions; radionuclide theranostics, combining diagnosis and therapy. Apparent is the dependency on source type, configuration and collimation, the emissions interacting in tissue, thereby depositing dose. Of particular note are the several advances in dose delivery that are placing ever increasing demands on the systems of dosimetry; consider for instance accommodating the spatial resolution needs of say small field in vivo dosimetry in an aqueous medium at high dose-rates. The glassbased TL systems offer attractions that include the robust nature of the medium, their impervious nature both to water and the EMF of MRIguided linacs, also acknowledging the high spatial resolutions that can be attained.

In the therapeutic regime, discrepancy in radiation delivered to the target can have highly adverse effects, the QA responsibilities being obvious. In this and other examples within radiation medicine, there is continuing need for enhanced accuracy and precision in the delivery and measurement of the optimum radiation dose. As with any other form of radiation dosimetry, developments towards utilising the luminescence yield of irradiated glass entails quantitative methods in determining the energy deposited within the medium (Attix, 1986).

\section{Choices in dosimetry}

In regard to the choices confronting the user, the many possibilities are far too great to cover in detail in any one short review. However, the more striking issues can best be illustrated through consideration of the most prominent of radiation monitoring devices, namely the ionization chamber (IC). The IC, regarded as the 'gold standard' quality assurance (QA) instrument throughout the realm of radiation medicine, utilizes relatively high voltages (i.e. several hundred or more), precluding invivo dosimetry. Additionally there is reliance on dose ionization conversion factors, also acknowledging the need to match energy transport (absorptive as well as scattering) within the dosimeter with that in the tissues of interest, a matter that has lead to Bragg-Gray Cavity Theory (and subsequent revisions to this). The IC and its calibration need to match the conditions that arise from this, giving route to the determination of absorbed dose in water from a measurement made in the detector. Particular demands to be satisfied are:

- That the cavity (the spatial dimension of the dosimeter) be small compared to the range of incident charged particles, the need being

\footnotetext{
* Corresponding author. Centre for Biomedical Physics, Sunway University, 50603, Kuala Lumpur, Malaysia.

E-mail address: d.a.bradley@surrey.ac.uk (D.A. Bradley).
} 